\title{
DAS INSTITUT FÜR IBEROAMERIKA-KUNDE
}

Die intensive Beschäftigung mit den Ländern Lateinamerikas und die Pflege der deutsch-lateinamerikanischen Beziehungen haben in Hamburg schon eine lange Tradition. Die bereits während der kolonialen Epoche des amerikanischen Subkontinents einsetzenden Handelsbeziehungen sind nach seiner Unabhängigkeit durch Handelsverträge und den Austausch von Konsulaten zwischen der Hansestadt und einigen der neuen Staaten erheblich verstärkt worden. Gleichsam als Nebenprodukt der kommerziellen Beziehungen ist dabei allmählich in Hamburg ein breites Fundament an Kenntnissen über die lateinamerikanischen Länder entstanden, verbunden mit der Bereitschaft, sich auch kulturell und wissenschaftlich mit diesem Teil der Neuen Welt zu beschäftigen. Daß dabei auch die ehemaligen iberischen Mutterländer Spanien und Portugal mitverstanden werden sollten, zeigt der Begriff „Ibero-Amerika“ im Namen derjenigen Institutionen, die im Laufe der Jahre als Träger dieser Arbeit entstanden sind.

Im Jahre 1916 wurde durch Initiative des Hamburger Romanisten Bernhard Schädel der „Hamburgische-Iberoamerikanische Verein “ gegründet. Ein Jahr darauf entstand das Ibero-Amerikanische Institut, dessen Leitung zunächst von Schädel und nach dessen Tod von Rudolf Großmann übernommen wurde. Das Institut, das bis 1923 ausschließlich aus privaten Mitteln, danach mit Hilfe eines Zuschusses der Freien und Hansestadt Hamburg finanziert wurde, widmete sich in zahlreichen Arbeiten der angewandten Forschung über Lateinamerika. Im Jahre 1945 wurde es als „Ibero-amerikanisches Forschungsinstitut der Universität Hamburg“ dem Romanischen Seminar der Philosophischen Fakultät angegliedert. Der 1916 gegründete Verein bestand in seiner ursprünglichen Form bis 1923, zuletzt unter dem Namen "Ibero-Amerikanische Gesellschaft". Es folgte ihm die "Gesellschaft der Freunde des Ibero-Amerikanischen Instituts“, die 1949 in dem heutigen IberoAmerika Verein aufging. Der Einsicht, daß die Wiederaufnahme der Handelsbeziehungen zu Lateinamerika nach dem zweiten Weltkrieg von einer verstärkten Pflege der kulturellen Kontakte begleitet sein müsse, entsprang im Jahre 1953 die Gründung der „Deutschen Ibero-Amerika Stiftung“, die seit ihrem Bestehen aus Spenden der deutschen Wirtschaft eine große Zahl von Forschungsstipendien an lateinamerikanische und deutsche Wissenschaftler vergeben konnte.

Mit der Erkenntnis der Problematik der Entwicklungsländer und im Verlauf der allmählich einsetzenden Entwicklungshilfe wurden in Deutschland der Mangel an fundierten Untersuchungen über die aktuellen Fragen Lateinamerikas und überhaupt die unzulängliche wissenschaftliche Beschäftigung mit diesen Ländern immer offenkundiger. Soweit an den deutschen Universitäten LateinamerikaForschung betrieben wurde, standen seit jeher die auf eine Erforschung der Vergangenheit sowie der natürlichen Verhältnisse gerichteten Disziplinen im Vordergrund. Die auf Lateinamerika bezogene Regionalforschung war, sieht man von den romanistischen Studien ab, fast ausschließlich eine Domäne der Archäologen, Ethnologen, Historiker und Geographen sowie der Zoologen und Botaniker. Für die Wirtschafts- und Sozialwissenschaftler war der Subkontinent als Forschungsobjekt weitgehend unbekannt. Die auf Initiative des Ibero-Amerika Vereins und der Deutschen Ibero-Amerika Stiftung erfolgte Gründung des „Instituts für Iberoamerika-Kunde" im Jahre 1962 hatte daher einen doppelten Zweck: durch wissenschaftlich fundierte Untersuchungen die Unterrichtung der deutschen Wirtschaftspraxis sowie der Außenpolitik über Gegenwartsfragen Lateinamerikas zu verbessern und zum anderen der wirtschafts- und sozialwissenschaftlichen Forschung und Lehre Anregungen für eine intensivere Beschäftigung mit dieser Region zu geben. Dabei strebte das Institut von Anfang an nach interdisziplinärer Zusammenarbeit. Die wissenschaftliche Durchdringung einer überseeischen Region unter Berücksichtigung ihrer historischen, geographischen, ethnologischen und sozio- 
ökonomischen Bedingungen geht über das Erfahrungsobjekt einzelner Fachdisziplinen weit hinaus. Das gilt in noch stärkerem Maße für alle Bemühungen, mit wissenschaftlicher Genauigkeit diejenigen politischen, sozialen und wirtschaftlichen Strukturänderungen zu begreifen, die gegenwärtig unter dem Begriff des Entwicklungsprozesses zusammengefaßt werden. In der traditionellen Konzeption der deutschen Wissenschaftsorganisation hat eine solchermaßen verstandene Regionalforschung wenig Raum. Von wenigen neueren Ansätzen abgesehen, ist die Lateinamerika-Forschung an deutschen Hochschulen noch immer einzelwissenschaftlich orientiert. Aus diesem Grunde fällt den nicht an eine Universität gebundenen Regionalinstituten eine wichtige Aufgabe zu. Mit dem Blick auf die komplexen Strukturen ihrer jeweiligen Region müssen sie versuchen, möglichst die Gesamtheit der für ihre Beurteilung relevanten Erkenntnisse zu gewinnen und eine weniger am Fachgebiet als am einzelnen Phänomen, wie z. B. der sozio-ökonomischen Entwicklung, ausgerichtete Forschung betreiben.

Das „Institut für Iberoamerika-Kunde" bemüht sich seit seiner Gründung, in Zusammenarbeit sowohl mit anderen deutschen Lateinamerika-Instituten als auch mit seinen Hamburger Schwester-Instituten in der "Stiftung Deutsches ÜberseeInstitut" in dem dargelegten Sinne seinen Beitrag zu einer zeitgemäßen und praxisnahen Lateinamerika-Forschung zu leisten. Sein Forschungsprogramm zeigt eine breite thematische Streuung. Wie aus den bisherigen Publikationen und den in Arbeit befindlichen Untersuchungen hervorgeht, überspannt es einen weiten Bogen von historisch-politischen Themen über geographische Untersuchungen bis hin zu aktuellen soziologischen und wirtschaftswissenschaftlichen Fragestellungen. Die dem Institut bei seiner Gründung gegebene Leitlinie, vorwiegend aktuelle und praxisnahe bzw. angewandte Forschung zu betreiben, liegt dabei allen Arbeiten zu Grunde. Leider hat es die bisher zahlenmäßig noch geringe personelle Ausstattung des Instituts nicht erlaubt, einen festen Mitarbeiterstab aufzubauen, in welchem alle in Betracht kommenden Fachgebiete ausreichend vertreten sind. Das Institut ist daher darauf angewiesen, einen Teil seiner Forschungsvorhaben an freie Mitarbeiter zu vergeben, obwohl auf diese Weise den Bedürfnissen einer interdisziplinären Koordination nicht immer Rechnung getragen werden kann.

Das in die Rechtsform einer Stiftung des bürgerlichen Rechts gekleidete „Institut für Iberoamerika-Kunde" ist in seiner Organisation und in seinen Funktionen eng mit dem Ibero-Amerika Verein und der Deutschen Ibero-Amerika Stiftung verbunden. Es untersteht der Aufsicht des Kuratoriums, das auch diese Stiftung beaufsichtigt. Vorsitzender des aus Persönlichkeiten der Wissenschaft und der Wirtschaft gebildeten Vorstands ist seit Gründung des Instituts der em. o. Professor Rudolf Großmann, früherer Direktor des „Ibero-amerikanischen Forschungsinstituts der Universität Hamburg“. Sein Stellvertreter ist der gegenwärtige Vorsitzende der Deutschen Ibero-Amerika Stiftung, Helmut Lorenz-Meyer. Die enge Zusammenarbeit zwischen der Institutsleitung und der Geschäftsführung des IberoAmerika Vereins hat sich seit der Gründung des Instituts ständig bewährt. Die praktische Arbeit des Vereins, die dabei in vielen Jahren gesammelten Erfahrungen befruchten die Tätigkeit des Instituts und geben ihr immer wieder neue Anregungen, während umgekehrt die Einrichtungen des Instituts, wie Bibliothek, Archiv und Dokumentation, ebenso wie die Ergebnisse seiner Forschungsarbeiten dem Verein bei der Bewältigung seiner Aufgaben zu helfen vermögen.

In den ersten beiden Jahren seines Bestehens wurde das Institut allein aus Spendenmitteln der „Deutschen Ibero-Amerika Stiftung“ getragen. Seit 1965 ist es mit den anderen drei Hamburger Regionalinstituten in der Stiftung „Deutsches ÚberseeInstitut" zusammengeschlossen und erhält über diese seine Basisfinanzierung aus Mitteln des Hamburger Staatshaushaltes. Von der Stiftung Volkswagenwerk erhielt das Institut, größtenteils über die „Arbeitsgemeinschaft Deutsche LateinamerikaForschung", beträchtliche Starthilfen für seine bibliographischen und dokumentarischen Vorhaben. 
Dem Institut gehören zur Zeit 8 wissenschaftliche und 12 andere Mitarbeiter (Bibliothekare, Dokumentare, Sachbearbeiter und Schreibkräfte) an. Darüber hinaus sind ständig 6 bis 8 freie wissenschaftliche Mitarbeiter mit Forschungsaufträgen des Instituts beschäftigt. Der wissenschaftliche Sachapparat des Instituts besteht aus einer Handbibliothek mit (Anfang 1968) rund 3000 Bänden, einem Zentralkatalog über das in fast 20 Hamburger Bibliotheken vorhandene LateinamerikaSchrifttum, einer Dokumentationsstelle und einem Archiv von Zeitungsausschnitten. Beim Ausbau seiner eigenen Bibliothek beschränkt sich das Institut bewußt auf die Anschaffung neuerer und aktueller Literatur zu den fachlichen Schwerpunkten seiner Forschungsarbeit. Dagegen weist der in einen Autoren- und einen Sachkatalog gegliederte Zentralkatalog schon jetzt über 100000 einschlägige Buchtitel in den verschiedenen wissenschaftlichen Bibliotheken Hamburgs nach. Die bibliographischen Arbeiten, die ihren Niederschlag in der Veröffentlichungsreihe "Bibliographie und Dokumentation" gefunden haben, sowie der Aufbau des Zentralkatalogs und das Informationsarchiv bilden die Basis für ein umfangreiches Dokumentationswesen, das innerhalb der Institutstätigkeit eine erhebliche Bedeutung erlangt hat.

Die „Arbeitsgemeinschaft Deutsche Lateinamerika-Forschung“ wählte im vergangenen Jahr das Hamburger Institut zu ihrem Dokumentationszentrum und beauftragte es mit dem Aufbau einer Dokumentationsleitstelle. Ihre Funktion ist es, in enger Zusammenarbeit mit den Mitgliedsinstituten der Arbeitsgemeinschaft und allen anderen dafür in Betracht kommenden Stellen wissenschaftlich relevante Publikationen sowie bestimmte andere Dokumente zu erfassen, auszuwerten und zugänglich zu machen. Der Aufbau der Leitstelle erfolgt in methodischer Abstimmung mit den anderen drei Hamburger Regionalinstituten, die ähnliche Dokumentationsstellen einrichten, sowie in Zusammenarbeit mit den daran interessierten Institutionen in der Bundesrepublik und im Ausland.

Überblickt man die bisherige Forschungstätigkeit des Instituts für IberoamerikaKunde anhand seiner Publikationen und der in Arbeit befindlichen Studien, so erkennt man eine Reihe thematischer Schwerpunkte. An erster Stelle stehen Themen, die sich mit der eigenständigen Entwicklung Lateinamerikas als Gesamtheit und mit seiner politischen und wirtschaftlichen Integration befassen. In diesen Bereich fallen die Studien, die sich beispielsweise mit der Organisation Amerikanischer Staaten (Heft 2 der SCHRIFTENREIHE), den Ansätzen zu einem gemeinsamen lateinamerikanischen Markt (Heft 3 und 4) und seinen verkehrsgeographischen Schwierigkeiten (Heft 5) befassen. Auch die Arbeiten über Bevölkerungsprobleme (Heft 7) und über bestimmte Rassenfragen (Heft 6), die der sozialen Integration des Subkontinents oder einzelner Länder entgegenstehen, gehören zu diesem Themenkreis. Einen zweiten Schwerpunkt bilden die Untersuchungen über Fragen der Entwicklungsfinanzierung. Im einzelnen handelt es sich dabei um Arbeiten über Fragen der Exportfinanzierung (Heft 8), über die ausländische Verschuldung Lateinamerikas (in Vorbereitung), über die Interamerikanische Entwicklungsbank (im Druck), über die staatlichen und privaten Entwicklungsgesellschaften einzelner Länder (in Arbeit) sowie über die Rolle der privaten ausländischen Direktinvestitionen in Argentinien, Brasilien México und Perú (z. T. abgeschlossen, z. T. in Arbeit).

Ein wesentlicher Gesichtspunkt ist bei diesen Untersuchungen, namentlich bei den Arbeiten über die ausländische Verschuldung und über Auslandsinvestitionen, das Verhältnis zwischen Lateinamerika und den Industrieländern, insbesondere Europas. Als Bezieher von staatlicher Kapitalhilfe und privatem Anlagekapital aus den Industrieländern nimmt Lateinamerika eine Sonderstellung unter den Entwicklungsregionen der Welt ein. Von besonderem Interesse für die Bundesrepublik ist die Gestaltung der Beziehungen zwischen der Europäischen Wirtschaftsgemeinschaft und Lateinamerika. Mit diesem Thema befaßte sich schon eine Untersuchung am Beginn der Institutstätigkeit (Heft 1). 
Den dritten Schwerpunkt bildet eine Reihe von Untersuchungen, die alle ein einzelnes Land, nämlich México, zum Gegenstand haben und bei unterschiedlichen fachlichen Ansätzen im Grunde das gleiche Phänomen, nämlich die politische und wirtschaftliche Sonderstellung Méxicos innerhalb Lateinamerikas, behandeln. Die politische Stabilität dieses Landes wird einmal unter historischen und verfassungsrechtlichen Aspekten - Revolution und Verfassung - (Arbeit im Druck), zum anderen anhand einer Analyse der mexikanischen Staats(Revolutions-)partei (Arbeit in Vorbereitung) untersucht. Fragen der wirtschaftlichen Stabilität und des wirtschaftlichen Nationalismus werden in der Arbeit über das Investitionsklima und die Lage der ausländischen Privatinvestitionen in México (im Druck) behandelt. Eine weitere Studie zum Rahmenthema México befaßt sich mit dem Problem: staatliche oder privatwirtschaftliche Initiative zur gewerblichen Berufsausbildung (in Arbeit). Die innerlich zusammenhängenden Arbeiten geben Gelegenheit zur Erprobung interdisziplinärer Forschungsansätze. An ihnen wurde auch besonders deutlich, wie wichtig bei einer gegenwartsbezogenen Regionalforschung die Zusammenarbeit mit in- und ausländischen Fachinstituten ist.

Die Bemühungen des Instituts um eine intensivere Zusammenarbeit mit den dafür in Betracht kommenden Institutionen richtet sich im inländischen Bereich an drei Gruppen von Einrichtungen: die in der "Arbeitsgemeinschaft Deutsche Lateinamerika-Forschung“ zusammengeschlossenen Institute; die durch die „Stiftung Deutsches Übersee-Institut" verbundenen anderen drei Regionalinstitute und schließlich die im Bereich der deutschen Außen- und Entwicklungspolitik tätigen Bundesbehörden, Stiftungen und anderen Institutionen. Mit den Mitgliedsinstituten und Einzelmitgliedern der Arbeitsgemeinschaft besteht vor allem ein Schriftenaustausch sowie in Einzelfällen eine Zusammenarbeit bei Forschungsvorhaben. Mit den Schwesterinstituten in der Stiftung Deutsches Übersee-Institut vollzieht sich die Zusammenarbeit hauptsächlich im Bereich des Dokumentationswesens, aber auch in grundsätzlichen thematischen und methodischen Fragen der Regional- und Entwicklungsländerforschung. Die Mitwirkung bei der Lösung von Fragen der deutschen Entwicklungspolitik geschieht durch Übernahme von Forschungsaufträgen, Bereitstellung von Informationsmaterial, Zusammenarbeit auf dem Gebiet des Dokumentationswesens und der Vorbereitung von deutschen Fachkräften für Aufgaben der deutschen Entwicklungshilfe für Lateinamerika.

Bei der Zusammenarbeit mit Forschungseinrichtungen in verschiedenen lateinamerikanischen Ländern geht es dem Institut vor allem darum, Partnerinstitute zu finden, mit denen gemeinsame Forschungsvorhaben vereinbart und durchgeführt werden können. Es soll sich dabei um Institute handeln, die zugleich den Angehörigen und freien Mitarbeitern des „Instituts für Iberoamerika-Kunde“ als Stützpunkte bei Feldforschungen dienen können. Es gehört zu den Grundsätzen des Instituts, Feldforschungen in Lateinamerika in möglichst enger Zusammenarbeit mit qualifizierten einheimischen Forschungsinstituten durchzuführen. Der entsandte Wissenschaftler soll, wenn irgend möglich, während seines Aufenthaltes im Lande als Gastforscher einem dafür in Betracht kommenden Institut eingegliedert werden. Die Erfahrung hat gezeigt, daß zumindest im Bereich der Sozialwissenschaften Forschungsarbeiten über Lateinamerika ohne engen Kontakt mit einheimischen Instituten und Wissenschaftlern nicht mehr erfolgreich durchgeführt werden können.

Von den Instituten, mit denen das „Institut für Iberoamerika-Kunde“ bisher in der geschilderten Weise zusammenarbeitet, ist das COLEGIO DE MEXICO in Mexico City hervorzuheben. Bis jetzt waren vier Mitarbeiter des Instituts dort als Gastforscher tätig. Die erwähnten Arbeiten über aktuelle Fragen der mexikanischen Politik und Wirtschaft wären ohne die enge Zusammenarbeit mit diesem angesehenen Forschungsinstitut kaum denkbar. Auch mit wissenschaftlichen Instituten in anderen Ländern hat sich eine ähnliche Zusammenarbeit angebahnt, so z. B. mit dem INSTITUTO TORCUATO DI TELLA in Buenos Aires. 
Die Publikationstätigkeit des Instituts und seiner Mitarbeiter erstreckt sich auf die Herausgabe einer Schriftenreihe, die Veröffentlichung einer Reihe „Bibliographie und Dokumentation" sowie auf Aufsätze in wissenschaftlichen und allgemein interessierenden Zeitschriften. Außerdem wirkt das Institut an dem Rundschreibendienst des Ibero-Amerika Vereins mit in Form aktueller Nachrichten über politische Fragen Lateinamerikas. In der Schriftenreihe (s. Anhang) sind bis jetzt 8 Hefte erschienen; die Reihe „Bibliographie und Dokumentation“ umfaßt bisher 14 Bände. Mehrere Arbeiten sind im Druck.

Das „Institut für Iberoamerika-Kunde" bemüht sich, im Rahmen der deutschen und internationalen Zusammenarbeit auf dem Gebiet der Lateinamerika-Forschung die ihm angemessenen Funktionen zu erfüllen. Standort, Struktur und Arbeitsweise des Instituts geben ihm häufig die Rolle eines Vermittlers zwischen verschiedenen Disziplinen, Instituten und Forschern. Es kann dabei von den Erfahrungen profitieren, die in den über 50 Jahren des Bestehens auf Iberoamerika spezialisierter Institutionen in Hamburg gewonnen worden sind.

v. G.

\section{ANHANG}

VEROOFFENTLICHUNGEN

DES INSTITUTS FƯR IBEROAMERIKA-KUNDE

\section{SCHRIFTENREIHE}

(erscheint im Übersee-Verlag, Hamburg)

Heft 1 H.-J.Hartmann und J. Westphalen: Europa auf falschem Kurs? - Lateinamerikanische EWG-Sorgen (deutsche und spanische Ausgabe), 1963 (vergriffen)

Heft 2 O. C. Stoetzer: Panamerika - Idee und Wirklichkeit. Die Organisation der Amerikanischen Staaten, 1964

Heft 3 H. Giesecke: Zentralamerika und sein Gemeinsamer Markt, 1964

Heft 4 Fr. Wehner: Die Lateinamerikanische Freihandelsvereinigung (ALALC), 1965

Heft 5 F. Henning: Intrakontinentaler Verkehr in Lateinamerika, 1965

Heft 6 Edith Oppens: Die Entrechteten - Indianisches Schicksal in den Andenländern im Spiegel der Literatur, 1965

Heft 7 J. Westphalen: Bevölkerungsexplosion und Wirtschaftsentwicklung in Lateinamerika, 1966

Heft 8 H.-J. Hartmann: Lateinamerikas Exportfinanzierung im Wandel, 1966 Außerhalb der Schriftenreihe sind erschienen:

Anke Schulz-Meinen: Prenda con Registro - Das Mobiliarregisterpfandrecht nach argentinischem Recht, 1965

Karl M. Helbig: Die Wirtschaft Zentralamerikas - kartographisch dargestellt und erläutert, 1966

\section{REIHE „BIBLIOGRAPHIE UND DOKUMENTATION“}

Heft 1 Das Arbeits-, Sozial- und Investitionsrecht iberoamerikanischer Länder, Eine Auswahl deutscher und fremdsprachiger Veröffentlichungen, bearbeitet von J. Pätz, 1963

Heft 2 Deutschsprachige Literatur über Wirtschaftsfragen Iberoamerikas, bearbeitet von Dr. Irene Roesler, 1964 
Heft 4 Landwirtschaftliche Fragen Iberoamerikas, Eine Ubersicht über das neuere deutsch- und fremdsprachige Schrifttum, bearbeitet von Dr. H. Niederböster, 1964

Heft 5 Bibliographie zur Politik und Zeitgeschichte der iberoamerikanischen Länder, bearbeitet von J. Schaefer, 1965 (erweiterte Neufassung von Heft 3)

Heft 6 Schöne Literatur lateinamerikanischer Autoren, Eine Übersicht der deutschen Übersetzungen mit biographischen Angaben (Autoren- und Übersetzerverzeichnis), bearbeitet von D. Reichardt, 1965

Heft 7 Wirtschaftsgeologische Literatur über Iberoamerika. Eine Übersicht über das neuere deutsch- und fremdsprachige Schrifttum, bearbeitet von Prof. Dr. G. Bischoff und cand. geol. Fr. Renger, 1966

Heft 8 Landwirtschaftliche Fragen Iberoamerikas, Eine Auswahl neuerer Zeitschriftenaufsätze, bearbeitet von Dr. H. Niederböster, 1965

Heft 9 Das Bildungswesen in der Gesamtentwicklung Lateinamerikas, Ausgewählte neuere Literatur (deutsch-spanisches Schlagwortverzeichnis), bearbeitet von W. Grenz, 1966

Heft 10 Quellenverzeichnis zur Wirtschaftsstatistik Iberoamerikas, Ausgewählte neuere Veröffentlichungen, bearbeitet von Chr. Wilhelms und José Guilherme de Almeida Sedas, 1966

Heft 11 Wirtschaft und Entwicklung Lateinamerikas, Ausgewählte, neuere Literatur

I: Außenwirtschaft, bearbeitet von Hermann Sautter, 1967

II: Produktion (Bergbau, Landwirtschaft, Industrie), bearbeitet von Wolfgang D. Fromm, 1967

III: Gesamtwirtschaftliche Fragen, bearbeitet von Erwin Klein, 1968

Heft 12 Bibliographie zur Soziologie und Demographie Lateinamerikas, bearbeitet von María Teresa Klein, 1968 